\title{
Increased Organic Contamination found on Mobile Phones after touching it while using the toilet.
}

\author{
Jannie Szeto', Bobby Sidhu², Fred Shaw ${ }^{3}$ \\ 1 Lead Author, B. Tech Student, School of Health Sciences, British Columbia Institute of Technology, 3700 Willingdon Ave, Burnaby, BC V5G 3H2 \\ 2 Supervisor, School of Health Sciences, British Columbia Institute of Technology, 3700 Willingdon Ave, Burnaby, BC V5G 3H2 \\ 3 Contributor, School of Health Sciences, British Columbia Institute of Technology, 3700 Willingdon Ave, Burnaby, BC V5G 3H2
}

\begin{abstract}
Background: Mobile phones are considered as an indispensable handheld item in society today. Frequently used, these devices are a "high-touched" commodity. Previous research has demonstrated that E. coli and other environmental contamination are responsible for the contamination of mobile phones. This study will measure the level of contamination (or sanitation) of mobile phones at an educational institution.

Method: The Hygiena MicroSnap Coliform and E. coli Enrichment Swab and the Coliform and E. coli Detection Swabs were used to detect the presence (or absence) of E. coli and total coliforms on subjects' mobile phones. The Hygiena UltraSnap ATP Surface Test was used to detect levels of ATP. The SystemSURE Plus Luminometer generated readings in RLUs that determined the level of sanitation. In addition, each subject answered two questions regarding their gender and whether or not they have touched their phones while using the toilet within the past week.

Results: No presence of E. coli or total coliforms were detected (0 RLUs). A one-tailed paired T-test confirmed that the ATP levels sampled from participants that touched their phones while using the toilet within the past week was statistically significant $(\mathrm{P}=0.008390)$. A two-tailed paired T-test confirmed that ATP levels was not statistically significantly different between males and females.

Conclusions: Based on the results, touching mobile phones while using the toilet contributes to increased ATP levels found on mobile phones. There were no differences in ATP levels found between males and females. Future studies are required to confirm this.
\end{abstract}

Keywords: ATP; E. coli; mobile phone; toilet; cross-contamination; fecal-oral route; organic contamination

\section{INTRODUCTION}

Mobile phones have evolved from being large devices permanently installed in vehicles as car phones (Jagadeesan et al., 2013) to personal and indispensable handheld items. As society shifts towards the use of advanced technology, and in particular mobile phones, many do not give a second thought as to where they take their phones. While we are concerned with hand hygiene throughout the flu season, many individuals are not consciously aware of how often they touch their phones. Since mobile phones are classified as a convenient personal device, it is often referred to as "high-touched" objects. A study in the United Kingdom revealed one in six cellular devices contained the communicable disease agent, E. coli (Collins, 2011), which is often found in contaminated food and water. When an individual brings their mobile device to the toilet, touches the door handle or the stall locks and then touches their phone, there is a risk that pathogens may be deposited onto their phones. A common scenario like this one begs the question "how sanitary are the general public's mobile phones"? According to microbiologists, the heat generated by our phones and our bodies in combination with 
constant handling promotes a reservoir for pathogens (Jagadeesan et al., 2013). Moreover, societal reliance on mobile phones overlooks the health significance associated with the sanitation of personal phones. Research of contaminated mobile phones in health care settings has been conducted; however, larger scale studies in educational settings are lacking. This research project is aimed to provide further information to the general public on the sanitation and the contaminants found on the public's mobile phones within an educational institution.

\section{LITERATURE REVIEW}

\section{E. coli and other pathogens found on mobile phones}

Escherichia coli (E. coli) is a gram-negative microbe that can grow either with or without oxygen and is found in the digestive tracts of warm-blooded mammals. Its presence indicates fecal contamination since it is found in the gut of warm-blooded mammals. When found in contaminated water or food, it is a strong indication of indirect or direct contamination with fecal matter either from an animal or from a person. Not all E.coli strains are harmful - the main one of concern is $E$. coli 0157:H7 due to its pathogenic nature. Bloody diarrhea and kidney damage can be a result of this particular strain of E. coli (CDC, 2011).

Recently, there have been rising concerns regarding individuals taking personal mobile phones to toilets with them. Society is encouraging us to be more reliant on technology and the communication that is associated with it. Hence, this places us in a fast-paced technological society that permits us to bring personal mobile phones everywhere we go. It is convenient for an individual to talk and send instant messages wherever they go and one is not likely to leave their phone unattended while using public toilets.
In the "Study of the role of mobile phones in the transmission of hospital acquired infections", Angadi et al. isolated only Staphylococcus aureus on health care workers' (HCWs) hands and mobile phones. Humans being the only reservoir, $S$. aureus is a bacteria that can be found in the respiratory tract and on the skin. About 53percent of the mobile phones were found to harbor Methicillin-resistant Staphylococcus (MRSA), a drug-resistant strain of $S$. aureus that can be acquired in a hospital settings (Angadi et al., 2014). Akinyemi et al. also found that $S$. aureus has the highest rate of contamination on mobile phones in various groups of individuals including food vendors, teachers, students, public servers and health workers. Following $S$. aureus in descending order of contamination: Enterococcus feacalis, E. coli, K. pneumonia, Bacillus spp. and $P$. aeruginosa. Angadi et al. confirmed that other studies which sampled mobile phones of HCWs also included these particular pathogens. Thus, it has been demonstrated that pathogens can survive on mobile phones.

\section{Fecal-oral route}

When E. coli is found in our food source, a person may experience illness. As a result, when fecal matter is found to be present in food, an infection will occur by means of either direct or indirect transmission. A direct transmission occurs when a person directly ingests, inhales or is in direct contact with bodily fluids from another person. In the case of the fecal-oral route, a person will likely ingest fecal matter via person to person contact such as oral-sexual transmission.

Alternatively, indirect transmission involves an intermediate object. The intermediate object contributes to the transmission of pathogenic organisms. Air, food, water, vectors such as insects and inanimate objects can act as a medium to successfully transfer infectious microorganisms. 
To highlight the importance of a foodborne and/or waterborne transmission, a famous Canadian case study in Walkerton, Ontario involved a waterborne outbreak associated with a contaminated municipal water supply in May 2000. This massive outbreak resulted in seven deaths and over 2000 ill cases. The agents responsible for the outbreak were Escherichia coli 0157:H7 and Campylobacter jejuni making it one of Canada's worst E. coli 0157:H7 outbreak in history (CBC News, 2010). A faulty chlorination system and a well that was vulnerable to contamination from cattle manure from a nearby local farm were factors that contributed to this incident (Hrudey et al., 2002). Water was the medium for transmission in this case as the unchlorinated groundwater was directly contaminated. Evidently, the outbreak was a result of poor management in the maintenance of the Walkerton water supply for the users and concurrently a failure to control and contain the occurrence of the outbreak.

\section{Cross-contamination}

Cross-contamination occurs when a pathogenic agent is unintentionally transferred from one object to another. In this case, only indirect transmission is considered since crosscontamination would involve an intermediate object as part of the fecal-oral route. For instance, cutting vegetables in preparation for a salad and cutting up raw chicken breast for baking on the same cutting board introduces pathogens into the ready-to-eat food item. The cutting board, also known as the object that facilitates pathogenic transmission is now a breeding ground for the pathogens found in the raw chicken and the chances of cross-contamination of Salmonella spp. to the ready-to-eat salad is high.

Kramer et al. evaluated the length of time pathogens could survive on dry surfaces. Grampositive bacteria such as $S$. aureus, Enterococcus spp. and $S$. pyogenes can survive for months.
Gram-negative bacteria such as E. coli, Klebsiella spp., and Shigella spp. can also survive for months on fomites. Consequently, mobile phones are at risk for contamination.

\section{BC Guidelines for Total Coliforms and $E$. coli}

Since $E$. coli is abundant in feces, it is used as an indicator of fecal contamination. Total coliform is an indicator for fecal and environmental contamination. As an indicator organism, the Food Quality Check Program Manual: Microbiological Recommendations from the BC Public Health Microbiology \& Reference Laboratory (Table 1) set the limits on less than 3 E.coli MPN/g as satisfactory and anything 3 or greater MPN/g of E. coli as unsatisfactory.

Table 1: Microbiological Recommendations for Ready-to-eat Foods

\begin{tabular}{|c|c|c|c|}
\hline \multicolumn{4}{|c|}{ Indicator Organisms (MPN/g) } \\
\hline Test & Satisfactory & Cautionary & Unsatisfactory \\
\hline Total Coliforms & $<100$ & $<1,000$ & $\geq 1,000$ \\
\hline Fecal Coliforms & $<3$ & $\mathrm{~N} / \mathrm{A}$ & $\geq 3^{*}$ \\
\hline E. coli & $<3$ & $N / A$ & $\geq 3$ \\
\hline
\end{tabular}

Schedule A in the Water Quality Standards for Potable Water in the Drinking Water Protection Regulation under the Drinking Water Protection Act prescribes no detectable E. coli or fecal coliform bacteria per $100 \mathrm{ml}$ sample of potable drinking water.

These guidelines emphasize how much $E$. coli and total coliform are regulated in the province's drinking water supply and food processing facilities. Hence, the presence of these indicator organisms in the environment is a public health concern.

\section{Mobile phones as fomites}

Fomites are contaminated inanimate objects and act as reservoirs for pathogens; therefore, mobile phones are classified as fomites. They have the 
ability to transmit pathogens indirectly through various modes of transmission such as ingestion (Lopez et al., 2014) and play a major role in the fecal-oral route.

An Egyptian study conducted by Hassan et. al discovered that enteric parasites could be transferred indirectly from person-to-person from contaminated currency coins and banknotes. As fomites, currency coins and banknotes are likely to contribute to a parasitic infection to exposed individuals. The lack of proper hand washing between handling money and the preparation of ready-to-eat foods contributed to the risk of cross-contamination. A common cultural behavior amongst the Egyptian population observed in the study was the wetting of the fingers with saliva while counting banknotes and handling food without properly washing their hands. As a result, currency circulation resulted in the increased risk of cross-contamination. Moreover, their findings also showed that mutilated banknotes with greater handling were far more contaminated than the fresh mint ones (Hassan et al., 2011).

Subsequently, because mobile phones are fomites, most of the research conducted on mobile phones and the various contaminants associated with them has been performed within a health care setting. A majority of nosocomial and foodborne pathogens can survive on fomites for several weeks and can linger on the fingers for hours (Lopez et al., 2014). Ninety percent of HCWs' hands and mobile phones were found to be contaminated with pathogens that resulted in nosocomial infections in Angadi et al.'s study. Similarly, Ustun and Cihangiroglu found that a rate of $97.8 \%$ of $\mathrm{HCWs}$ ' mobile phones were found to be contaminated with Methicillinresistant Staphylococcus aureus (MRSA) and E. coli. A lack of awareness about mobile phones being a fomite and nosocomial infections in general explains the reason for this (Ustun \& Cihangiroglu, 2012). Tekerekoğlu et al. conducted a cross-sectional study, also in a health care setting, found the same pathogens to be present on mobile phones of patients, patient's companions, visitors and HCWs. Since phones from the cross-sectional study sample groups mentioned revealed evidence of contamination, phones are a potential cause of hospitalassociated infections. Furthermore, there is a high possibility that pathogens were introduced to the hospital from the environment as visitors' phones were tested. This would mean that there is an increased chance that students' and teachers' mobile phones at a public educational setting will be contaminated. The findings within these studies were all consistent: mobile phones used in a health care setting were found to be contaminated with disease-causing microorganism.

\section{Adenosine Triphosphate}

Adenosine triphosphate (ATP) is found in all living things and things that were once alive. Since this energy molecule is found in all cells, it is a general biological indicator (Hygiena, 2014d). ATP is found in foods, pollen from plants and even the sweat on an individual's palm. ATP swabs are used in detection of levels of surface biological contamination industries such as in food production and food service industries, pharmaceutical manufacturers and hospital settings. Moreover, the tests are used widely as they offer real-time, feasible, rapid and quantifiable readings when measuring the effectiveness of cleaning (Richard et al., 2013). Since mobile phones are classified as an indispensable handheld item, biological contamination is presumably present on the surface of these devices. ATP does not directly measure the presence (or absence) of a particular species of bacteria or virus of interest; however, it is a reliable method for the detection of contaminants of biological origin (Shaughnessy et al., 2013). Thus, ATP surface testing on mobile 
phones will demonstrate the sanitary condition as they are a concern for cross-contamination.

\section{EHO involvement: control measures}

Environmental health officers (EHOs) are responsible in providing education and advice on sources of contamination and proper hand washing, especially growing with concerns of contaminated fomites in society due to inadequate hand hygiene. Mobile phones are personal items that are carried around by individuals in society on a daily basis. They are constantly being touched since they serve multiple functions (Jagadeesan et al., 2013). An individual can mistakenly bring their mobile phone to the toilet due to the sake of convenience. There are further reasons as to why mobile phones serve as a reservoir for microorganisms. First, heat is generated by the phone when the phone is turned on. Second, storing phones in pant pockets as well as leaving them in purses can act as an incubator allowing pathogens to thrive in these environments (Jagadeesan et al., 2013). When a fomite is touched prior to a meal, the pathogen has a greater chance of being ingested. This is an example of a classic fecal-oral route by means of contamination of mobile phones. In preventing the spread of infectious diseases, it is important for one to recognize the importance of environmental factors and hand hygiene in places such as in the home, public areas, and health care facilities (Lopez et al., 2014). Consequently, the role of the EHO is to provide awareness on fomite contamination and education on appropriate hand washing and hand hygiene.

An example of determining the movement of viral contamination by using fomites as indicators lies within Sifuentes et al.'s study. The spread of viruses from one contaminated hotel room to another by hotel housekeepers was observed and evaluated as well as the spread of viruses from guests that participated at a conference at the same hotel. Even with the appropriate hygiene intervention implemented while the study was being performed, they managed to reduce the spread of viruses simply by providing hand hygiene products and disinfecting wipes to the housekeeping guests and staff. The study concludes with a hygiene intervention program in place, the spread of viruses between the groups were greatly reduced (Sifuentes et al., 2014). By implementing a hygiene intervention program, EHOs can facilitate in educating the public about hand hygiene and the importance of sanitizing their phones.

In Angadi et al.'s study about isolating $S$. aureus from HCWs' mobile phones and hands and resulting in nosocomial infections, some control measures were suggested. Strict infection control practices as well as using an alcohol sanitizing protocol of the mobile devices should be used to control the spread of pathogens within a health care setting (Angadi et al., 2014). Again, hand hygiene is the main intervention that is recommended by the literature (Srikanth et al., 2010).

Donofrio et al. found various hotspots for pathogenic growth within the "high-touched" areas of the house. Aside from the high microbial concentration found in the kitchen, the authors also considered personal items such as mobile phones and laptop keyboards and found that there were microbial counts present on them as well. They recommended frequent sanitizing of surfaces as they can potentially lead to foodborne illnesses (Donofrio et al., 2012). In addition, they mentioned that their findings would be useful to environmental health professionals in educating the public, performing risk assessments, implementing appropriate measures for prevention and to establish appropriate guidelines involving household hygiene.

Finally, a review of recent studies reported on contaminated mobile devices in a health care setting found 9-25 percent were contaminated with pathogens (Brady et al., 2009). Other than 
focusing on decontaminating the device's surface, as this can cause malfunction of the device (Collins, 2011), the review suggested recommendations to reduce contamination risks. Educating the staff, implementation of a strict hand hygiene protocol, having guidelines on device cleaning and restricting use of mobile phones in high risk areas in a health care setting would aid in the prevention of nosocomial infections (Brady et al., 2009). Aside from establishing protocols at a hospital setting, the recommendations can be directed towards the general public. The EHOs' involvement in this is vast. Education would be the most effective way in communicating with the public about the importance of proper hand washing and increasing the awareness factor of the sanitation of their indispensable device. As advocates for public health, EHOs can be engaged in health promotion as a way to provide further information to the public.

\section{RESEARCH QUESTION}

Based upon the various literature that has been done regarding contamination of mobile phones, this raises the question of what is the sanitation status of personal mobile devices. Mobile phones can play a major role in the fecal-oral route in the transmission of communicable diseases. E. coli is a concern due to it being a fecal matter indicator and the health significance associated with it. The societal shift towards popular use of technology requires one to be attentive towards their mobile devices. As these handheld devices are an indispensable item to many individuals, even while using the toilets, we can expect an increased rate of contamination upon these fomites. Many studies focused on mobile phones in a health care setting, but only one study used a group consisting of teachers' and students' mobile phones in an educational institution. Moreover, a person-to-person transmission typically involves poor hand washing practices after using the toilets (E. coli 0157:H7 Infection, 2006). The research conducted in this study will be of interest to any environmental health professional including EHOs as it involves education and increase in awareness of hand hygiene. The purpose of this research project was to determine the level of sanitation by means of the detection of Adenosine Triphosphate (ATP) and the presence (or absence) of E. coli on the mobile phones at an educational institution. To address this, participants that touched their phones while using the toilet in the past week were compared to the ones that did not touch their phones and whether there is a difference. In addition, males' and females' phones were analyzed for difference in sanitation.

\section{MATERIALS AND METHODS}

\section{Materials}

A complete list of materials and the corresponding description of the materials used for this study is outlined in Table

Table 2: List of materials used for this study.

\section{Materials}

Nitrile gloves

Subject's mobile phones

Hygiena UltraSnap ATP Surface Test

\section{Description}

To maintain an aseptic environment.

The samples for the study.

A pre-moistened swab equipped with a liquid-stable reagent that is used to perform an ATP surface sample test 


\begin{tabular}{|c|c|}
\hline Hygiena MicroSnap Enrichment Swab & $\begin{array}{l}\text { Contains a specific growth medium for } E \text {. coli and } \\
\text { coliform (Hygiena, 2014a) }\end{array}$ \\
\hline $\begin{array}{l}\text { Hygiena MicroSnap Coliform Detection } \\
\text { Device }\end{array}$ & $\begin{array}{l}\text { Contains a bioluminogenic substrate for coliform } \\
\text { detection (Hygiena, 2014a) }\end{array}$ \\
\hline SystemSURE Plus ${ }^{T M}$ Luminometer & $\begin{array}{l}\text { Detects the light emitted from the Hygiena } \\
\text { UltraSnap - ATP Surface Test and generates a } \\
\text { numerical reading in relative light units (RLUs) } \\
\text { (Hygiena, 2014d) }\end{array}$ \\
\hline Calibration Control Kit & $\begin{array}{l}\text { To further verify that the SystemSURE } P l u s^{T M} \text { is } \\
\text { within calibration. Contains a reusable positive and a } \\
\text { negative control rod for specification confirmation } \\
\text { (Hygiena, 2014d) }\end{array}$ \\
\hline Incubator & To incubate the swabs at $37^{\circ} \mathrm{C}$ \\
\hline Timer/Clock & To set the time for incubation \\
\hline Microfiber cloths & To wipe off subject's mobile phones after swabbing \\
\hline Empty tissue box & For subjects to drop off their anonymous responses \\
\hline $\begin{array}{l}\text { Office supplies: } \\
\text { Clipboard, paper, pen, ruler }\end{array}$ & To record findings \\
\hline NCSS software & For statistical manipulation. \\
\hline $\begin{array}{l}\text { Microsoft Office } 2013 \\
\text { Excel } \\
\text { Word }\end{array}$ & $\begin{array}{l}\text { To analyze descriptive data } \\
\text { To format and word process the article }\end{array}$ \\
\hline
\end{tabular}

\section{Standard methods}

The subjects' mobile phone samples were collected at the British Columbia Institute of Technology's (BCIT) Burnaby campus. Samples were collected in two building: building SWI and SE2. SW1 is where the main entrance to the school is located and is the home to Health Sciences programs offered at BCIT. In addition, the Registrar's Office, Student Information Services, Enrolment Services, Program Advising and other student services are all located at SW1 (BCIT, n.d.). Thirty-five mobile phone samples out of 68 samples were collected at the foyer in SW1 on December 12, 2014. On December 15, 2014, the remainder 33 samples were collected in the student association building, also known as SE2. SE2 is the heart of BCIT's Burnaby Campus. It comprises of a dining area, a bookstore and conferences and meeting rooms (BCIT, n.d.). Due to high student traffic within these two buildings, they were selected as venues for sampling. Hence, representative samples were collected as a result.

Every subject that agreed to offer their mobile phones for sampling via verbal consent were given two questions to answer. The two questions the subjects were asked to answer were the following: i.) what is your gender? ii.) In the past week, have you touched your phone while using the toilet? The responses to the questions were identified to the corresponding phone while remaining anonymous, the slip of paper with the two questions were numbered to the equivalent MicroSnap coliform and UltraSnap ATP swabs. The researcher prepared the swabs by labeling both swabs with a specific number.

When the subjects verbally agreed to have their personal phones sampled, the subjects were asked to fill out the questionnaire and to answer the two questions. Participants that were interested in being contacted with the results were to include 
their email at the back of the questionnaire. The questionnaires were collected in an empty tissue box. Phone samples were collected using the Hygiena MicroSnap Enrichment Swab containing a growth medium for coliforms and $E$. coli specifically and the Hygiena UltraSnap ATP Surface Test. The author numbered the questionnaire that corresponded both swabs.

As the subjects filled out the questionnaire, the author took a swab sample with the Hygiena MicroSnap Enrichment Swab on approximately half of the mobile phone's surface (front, sides and back of the device) by using the traditional swabbing method to get a representative sample. The traditional swabbing method used involved applying sufficient pressure while swabbing horizontally within the specified area, then vertically (perpendicular to the initial direction) and finally at a $45^{\circ}$ angle. The swab must be continuously rotated while swabbing (HygienaTV, 2013). The remaining half of the surface area of the device was swabbed using the Hygiena UltraSnap ATP Surface Test. After swabbing with the traditional method, each and every subject's mobile phone was wiped with a microfibre cloth to remove any residue from the swab.

The Enrichment swabs were incubated at $37^{\circ} \mathrm{C}$ for seven hours for the presence/absence of coliforms and E. coli (HygienaTV, 2013). Prior to incubation, swabs were activated by bending the bulb forward and backward to release the liquid-stable reagent. Squeezing the bulb will stimulate the liquid to flow to the bottom of the tube where the swab bud would bathe. The tubes were shaken gently to mix the sample in the liquid.

The UltraSnap ATP swabs did not require incubation; thus, it was activated and tested instantly. The ATP swabs were activated in the same manner as the Enrichment swabs. The tube was shaken gently for 5-10 seconds (Hygiena, 2013e). The Calibration Control Kit was used to confirm that the SystemSURE Plus luminometer was properly calibrated (HygienaTV, 2013). The luminometer was kept upright while the ATP swabs were inserted into the luminometer. After closing the lid, the measurement reading was initiated by pressing "OK". The SystemSURE Plus is programmed to conduct a 15 seconds calibration verification every time the device attempts a reading. The readings were recorded.

After the Enrichment swabs were incubated for seven hours, the contents were transferred to the detector tubes for coliform detection. The detector tubes was incubated after activating it in the same manner as the Enrichment tubes for another 10 minutes at $37^{\circ} \mathrm{C}$. The detector tubes were inserted into the luminometer for measurement. Each detection device contained a bioluminogenic substrate that allowed the luminometer to easily detect light that is produced when the enzyme from the pathogen of interest is present. The unit is measured in Relative Light Units or RLUs (Meighan, 2014). Table 2 outlines the threshold value for presence and absence of coliform and E. coli. When confirming presence of coliform (cfu/ml), 10 or more RLUs must be measured when using the SystemSURE ${ }^{\text {TM }}$ Plus.

Table 2: Presence/absence Threshold Value

Result SystemSURE Plus

\begin{tabular}{|l|c|}
\hline Absent & $0-9$ \\
\hline Present & $\geq 10$ \\
\hline
\end{tabular}

(Hygiena, 2013b)

\section{Reliability and Validity of Measures}

Reliability. The MicroSnap received a Performance Tested Method Validation from the AOAC Research Institute (Hygiena MicroSnap, 2014b). The sampling of the participants' mobile phones were all administered in the exact same order of the procedure using the same instruments and equipment. However, the variations of the size and the surface area available on the mobile phone were taken into consideration. Every 
tangible surface area of a phone was tested. Onehalf were tested using the MicroSnap Enrichment Swab and the remaining second half were tested with the ATP Surface Test swab - refer to the standard method section for further information. In addition, the sampling was all done by the researcher.

Validity. The Hygiena SystemSURE Plus ${ }^{\mathrm{TM}}$ luminometer have a field calibration installed in as the specifications of the device (Hygiena MicroSnap, 2014b). Furthermore, the detection device can be used for other systems such as the Surface ATP, Water ATP, Allergen Prevention, Alkaline Phosphatase, and Acid Phosphatase (Bassan et al., 2014). In terms of external validity of the study, the participants of the research provided samples at BCIT. In general, the method of this research and the result can be generalized to other educational institutions.

\section{Calibration of Instruments}

The SystemSURE Plus ${ }^{\mathrm{TM}}$ luminometer was the only instrument that required a calibration in this research. It has a built-in field calibration check. A 15 second countdown verifies the device's calibration status (Hygiena, 2014b).

The Calibration Control Kit included a positive and negative rod to ensure that the field calibration within the specifications are up-todate. These control systems are used in conjunction to verify the proper calibration of the unit. When inserted, the positive control rod must score between 45-85 RLUs and the negative control rod must score between 0-5 RLUs (Hygiena, 2013e).

\section{Inclusion and Exclusion Criteria}

Female and male subjects that did touch or did not touch their mobile phones while using the toilet were included in the data set. However, subjects that did not touch their mobile phones while using the toilet, but did take their phones with them into the washroom were excluded.
Thus, the researcher asked specifically whether or not they have touched their device while using the toilet in the past week as one of the two questions from the questionnaire.

\section{Ethical Considerations}

Verbal consent was given to the researcher from the participants of the study. The researcher explained the purpose of the study to every participant and what is required from them.

\section{Pilot Study}

A pilot study was conducted using the proposed standard method. Only four samples were used to perform the pilot study.

No E. coli was detected on the four mobile phones (0 RLUs). On the contrary, the luminometer detected 0-2 RLUs of coliform after leaving the Detection Device at room temperature for more than 10 minutes. With reference to Table 2, 0-2 RLUs coliform is considered as "absent". Nonetheless, the luminometer did generate a reading for coliform in comparison to detecting the presence of E. coli. Based on the results of the pilot study, the researcher decided on using the proposed standard method to conduct the study and excluded the detection of $E$. coli.

\section{RESULTS}

No total coliform was found to be present on subjects' mobile phones. Readings of 0 RLUs were generated from the MicroSnap Coliform Detection Device. As a result, the researcher decided to only test for ATP.

\section{Description of Data}

The research was conducted using a combination of continuous numeric and nominal dichotomous data. A short questionnaire that consisted of two questions was distributed to participants that offered their phones to be swabbed. The first question on the questionnaire was: what is your gender? The participants circled either " $F$ " for 
female or " $\mathrm{M}$ " for male. The second question was: in the past week, have you taken your mobile phone in the toilet and touched it at any point while you were there? The answer to the question was recorded as either "yes" or "no". The questionnaire delivered the nominal dichotomous data while the ATP reading from the SystemSURE Plus ${ }^{T M}$ Luminometer generated continuous numeric values in RLUs.

\section{Statistical Packages Used}

The author used NCSS 9: Statistical Analysis and Graphics to analyze the data and findings. Microsoft Office 2013: Excel 2013 was used to generate descriptive data and to illustrate the data in a chart and graph format.

\section{Descriptive Statistics}

The average ATP reading collected using the ATP Surface Test data at building SW1 and SE2 was 262 RLUs. The median, range and standard deviation was found to be 155, 1543 and 314 RLUs, respectively.

Of the 68 subjects, 34 males and 34 females participated in the research. Figure 1 illustrates that 45 percent of males and 55 percent of females reported touching their phone at least once within the past week while using the toilet. Sixty-two percent of the subjects reported touching their phone within the past week while using the toilet while 38 percent did not (Figure 2).

Figure 1: Percentage of Gender that touched their Phones while using the Toilet

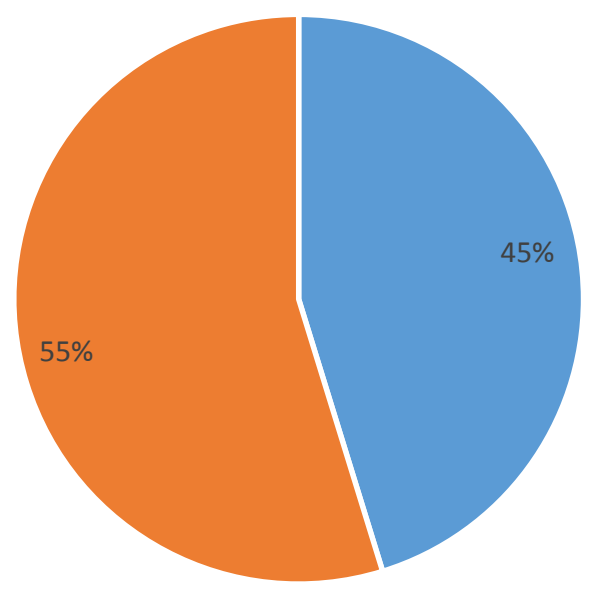

Figure 2: Percentage of Mobile Phone Handling

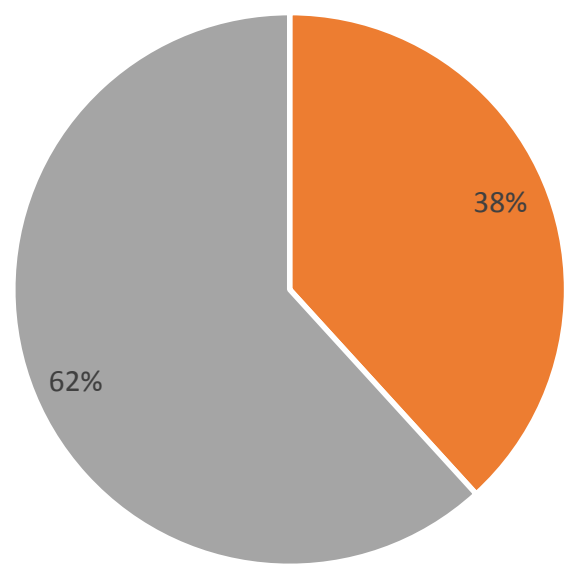

- Did not Touch Phone - Touched Phone

\section{Inferential Statistics}

Two independent-two-sampled T-tests were used to analyze the data collected. Two independent null hypothesis $\left(\mathrm{H}_{0}\right)$ and alternative hypothesis $\left(\mathrm{H}_{\mathrm{a}}\right)$ were generated. With support from the literature review and the results produced from the descriptive data, it was suggested that ATP levels are higher in individuals that touched their phone while using the toilet than those that did not. Hence, a two-sample-one-tailed T-test was used to produce the results. However, the assumption of not having the knowledge as to whether ATP levels are greater in females or males, a two-tailed T-test was used to determine whether there is a difference in ATP levels.

\section{Statistical Analysis}

Null Hypothesis $1\left(H_{o} 1\right)$ : There is no difference in ATP levels between participants that touched their phone while using the toilet in the past week and those that did not touch their phone $\left(\mu_{\text {Touched }}\right.$ $=\mu_{\text {Untouched }}$ ).

Alternative Hypothesis $1\left(H_{a} 1\right)$ : The ATP levels are greater in participants that touched their phone while using the toilet in the past week than 
those that did not touch their phone $\left(\mu_{\text {Touched }}\right)$ $\left.\mu_{\text {Untouched }}\right)$.

Interpretation of Results $\left(H_{o} l\right.$ and $\left.H_{a} l\right)$. From the tests of assumption, the Mann-Whitney U Test was used to interpret the findings. A onetailed T-test ( $\left.\mu_{\text {Touched }}>\mu_{\text {Untouched }}\right)$ with a p-value of 0.008390 indicated significance; thus, confirming that individuals that touched their phones while using the toilet within the past week scored higher ATP levels than those that did not touch their phones. The power of the test at $\alpha=$ 0.05 is 75 percent.

Null Hypothesis $2\left(H_{o} 2\right)$ : There is no difference in ATP levels between males and females $\left(\mu_{\text {males }}\right.$ $\left.=\mu_{\text {females }}\right)$.

Alternative Hypothesis $2\left(H_{a} 2\right)$ : There is a difference in ATP levels between males and females $\left(\mu_{\text {males }} \neq \mu_{\text {females }}\right)$.

Interpretation of Results $\left(H_{o} 2\right.$ and $\left.H_{a} 2\right)$. The Mann-Whitney U Test of assumption was used since the data was not normally distributed. It was found that $\mathrm{P}=0.892672$; therefore, $\mathrm{H}_{0} 2$ cannot be rejected. This indicated that there were no differences in ATP levels between males and females.

\section{DISCUSSION}

From previous research, mobile phones were demonstrated as fomites as they are subject to cross-contamination. Research indicated various pathogens present on mobile phones such as $E$. coli and S. aureus. Angadi et al. demonstrated that pathogens are present on both health care workers' hands and phones. In addition, half of the samples were found to harbor MRSA (Angadi et al., 2014). Thus, individuals that touched their phones while using the toilet are likely to contaminate their phones than those that did not touch their phones while using the toilet. Consistent findings were drawn based on the results from this study. The rejection of $\mathrm{H}_{0} 1$ due to a significant $\mathrm{p}$-value from the one-tailed t-test confirmed the persistent findings to previous research. Donofrio et al.'s revealed that hightouched areas generate higher levels of microbial counts. ATP is an indication of levels of organic contamination on a surface. Hence, deposits of organic material on phones occur when the device is touched. As a result, pathogenic growth is primarily present on high-touched surfaces (Donofrio et al., 2012); thus, yielding high levels of organic material. Aside from E.coli and total coliform, no other pathogens were tested. However, high levels of ATP suggested that there were biological forms of contaminants present including bacteria, fungus and viruses even when the ATP Surface Test did not directly test for them (Shaughnessy et al., 2013).

Table 3: Recommended Benchmark Limits for Hospitals

\begin{tabular}{|l|c|c|c|}
\hline Surface/Application & Pass & $\begin{array}{c}\text { Caution } \\
\text { Range }\end{array}$ & Fail \\
\hline Hospital Public Areas & $<50$ & $51-100$ & $101+$ \\
\hline Near patient areas & $<25$ & $26-50$ & $51+$ \\
\hline Sterile services & $<10$ & $11-30$ & $31+$ \\
\hline Washer disinfector & $<5$ & $6-10$ & $11+$ \\
\hline Food preparation/catering & $<10$ & $11-30$ & $31+$ \\
\hline
\end{tabular}

(Hygiena, 2013c)

Hygiena's ATP cleaning verification system provides benchmark limits for surface sanitation as recommendations to hospitals (Table 3). Since there are no specific recommendations provided by Hygiena regarding ATP benchmark limits for mobile phone surfaces, Table 3 can be used as a reference when interpreting appropriate levels of ATP found in this research. In addition, Hygiena established a pass or fail limit for health care workers' hand hygiene to be 60 RLUs (Hygiena, 2013d). According to the statistics generated, the participants' mobile phones were found to have an average of 262 RLUs with the median being 155 RLUs. However, table 3 generated by Hygiena (2013c) illustrates strict benchmark limits with the worst fail case being 101 RLUs 
and less than 50 RLUs is a pass. In reality, ATP levels found on mobile phones are significantly higher than the strict limits established for hospitals. When establishing hospital sanitation limits, the same limits cannot be realistically applied to public areas since hospitals deliberately sanitize frequently to protect the public from experiencing an infection due to contamination (Hygiena, 2013c). In the case of mobile phones being a high-touched item, it is likely to have additional ATP detected from the hands. ATP is naturally found in our skin and even in the sweat on our palms, thus it is not a threat to health (Hygiena, 2013d).

When collecting the data, the researcher recorded the number of individuals that were approached and asked to participate in the research but refused to. Throughout the two days of data collection at BCIT, 15 individuals refused to participate in the research. The reason varies and may include: individuals may be time constraint at the time of the invitation since it was examination period during that particular week; a lack of interest to participate; individuals may be embarrassed and did not want to disclose the results of the sanitation status of their device (F. Shaw, Personal communication, December 15, 2014).

\section{RECOMMENDATIONS}

Based on this study, the promotion of hand hygiene, awareness and maintaining a sanitary status on mobile phones is highly recommended at educational institutions. In addition to the signage and promotional posters in washrooms and in food preparation areas, it is important to promote awareness regarding mobile phones' ability to act as a reservoir for pathogens. As a potential source for contamination, phone usage in the washroom is a health concern. A promotional campaign could be organized by the institution which may involve the arrangement of an informational booth as part as a deliverable strategy to students and the public at the institution. Posters can be used remind students to sanitize their phones on a regular basis and not to use their phones while using the toilet. Finally, EHOs can use this information to educate the public on the importance of seeking appropriate levels of the sanitation on mobile phones. This can be achieved by utilizing certain means of deliverables as educational tools.

\section{LIMITATIONS}

Time and budgetary constraints were major limiting factors that contributed to the research. Within one week, the researcher collected all 68 samples from two buildings, incubated the samples, generated data for each of the samples and recorded the questionnaire data onto an Excel spreadsheet. If time was not a limiting factor, a larger sample could be collected throughout the BCIT campus instead of being restricted to only two buildings. This would have generated a more representative sample of the population within an educational setting. As for budget, the researcher was limited to using 68 UltraSnap ATP swabs, 68 MicroSnap Coliform and E.coli Enrichment Swab and 68 of the MicroSnap Detection Device. Since no coliform and E.coli were detected, all 136 swab samples were disregarded as the data served insignificant purposes while only 68 ATP UltraSnap swabs' data were analyzed and recorded. With a flexible budget, a greater number of swabs can be used to produce a larger sample size which will likely result in a statistically significant outcome when seeking a difference in ATP levels between males and females.

ATP is readily present in high-touched surfaces such as on mobile phones. When testing for levels of ATP on phones, it is expected to be detected by the luminometer. Organic contamination found on sampled phones was not entirely dependent on whether or not the individual touched their phones while using the toilet during 
the past week. Consequently, this does not demonstrate that contamination is directly from using the toilet. Poor hand hygiene is a prevailing indicator to the increased levels of ATP on fomites. Thus, contamination found on mobile phones after touching it while using the toilet is only a contributing factor to increased ATP levels.

E. coli and total coliform were not found to be present after testing 68 samples. Firstly, the sensitivity of the instrument is questionable at the time when detecting accurate ATP levels. Hence, human error shall be taken into consideration. Secondly, only half of the entire device was swabbed. In this case, the swab may not have collected a representative sample of the surface contamination. Phone sizes and the design differs as some are smaller or larger while some have a keyboard component that can fold and flip out. Relying only on visual judgment, the researcher swabbed roughly half of the device using the ATP UltraSnap and half using the Enrichment Device. Therefore, E. coli and total coliform may not have been collected. Another possible contributing limitation to the absence of the pathogen of interest is the public's common habit of constantly inserting mobile phones in pockets and purses after use. Pathogens can therefore transfer and deposit there.

\section{FUTURE RESEARCH}

To acquire a stronger linkage between pathogenic contamination on mobile phones and its usage while using the toilet is required. The following are suggestions for future research:

A larger sample size is required

Test for another indicator organism

$>$ Sample the entire phone with one test

Use a similar instrumentation of a different brand

Use an alternative method
A longer and extensive questionnaire is required:

$>$ The frequency of phone sanitization

The frequency of touching their phones while using the toilet

Average duration of phone use while using toilet

$>$ What program at the educational institution are the participants in

\section{CONCLUSIONS}

Organic contamination in this study is found to be higher amongst participants that touched their phones while using the toilet within the past week than those that did not. Performed at an educational setting, the findings of this study is consistent with previous research. However, the relationship between using mobile phones while using the toilet and contaminating it with pathogens such as E. coli was not established in this study. Phone usage in the washroom is only a contributing factor and thus, to establish a stronger representative relationship, a larger scale research with less time constraint and a flexible budget is required. On the other hand, there were no differences in ATP levels observed between males and females. Future studies could address the limiting factors to confirm the findings presented in this study.

\section{ACKNOWLEDGEMENTS}

The author would like to thank Fred Shaw for organizing and providing support throughout the entire data collection process and ordering the required equipment. The author is truly grateful to have Bobby Sidhu supervise the research and offering expert advice on conducting the study and writing this research paper. Lastly, the author would like to thank Helen Heacock for her suggestion on the research topic and advice on the study design. All their efforts and input into this research is greatly appreciated. 


\section{COMPETING INTEREST}

The authors declare that they have no competing interests.

\section{REFERENCES}

Akinyemi, K., Atapu, A., Adetona, O., \& Coker, A. (2009). The potential role of mobile phones in the spread of bacterial infections. The Journal of Infection in Developing Countries, 3(8), 628-632.

Angadi, K., Misra, R., Gupta, U., Jadhav, S., \& Sardar, M. (2014). Study of the role of mobile phones in the transmission of Hospital acquired infections. Medical Journal of Dr. D.Y. Patil University, 7(4), 435-438.

Bassan, A., Sidhu, B., Keilbart, K., \& Shaw, F. (2014). Determining the time required to disinfect a sponge contaminated with Escherichia coli, using a commercial microwave (pg. 1-12).

Brady, R., Verran, J., Damani, N., \& Gibb, A. (2009). Review of mobile communication devices as potential reservoirs of nosocomial pathogens. Journal of Hospital Infection, 71, 295-300. doi :10.1016/j.jhin.2008.12.009

British Columbia's Institute of Technology (BCIT). (n.d.). Life at BCIT: Student Life. Retrieved from http://www.bcit.ca/lifeatbcit/studentlife.shtml

Collins, E. (2011, October 11). One in six mobile phones carry E. coli. The Daily Telegraph. Retrieved October 15, 2014, from http://www.cbc.ca/news/health/1-in-6cellphones-have-fecal-e-coli-traces1.1120615

Donofrio, R., Bechanko, R., Hitt, N., O'Malley, K., Charnauski, T., Bestervelt, L., Saha, R., Saha, N. (2012). Are We Aware of Microbial
Hotspots in Our Household? Journal of Environmental Health, 75(2), 12-19.

E. coli 0157:H7 Infection. (2006, December 1). Retrieved October 16, 2014, from https://www.health.ny.gov/diseases/communi cable/e_coli/fact_sheet.htm

Hassan, A., Farouk, H., Hassanein, F., \& AbdulGhani, R. (2011). Currency As A Potential Environmental Vehicle For Transmitting Parasites Among Food-related Workers In Alexandria, Egypt. Transactions of the Royal Society of Tropical Medicine and Hygiene, 519-524. doi:10.1016/j.trstmh.2011.05.001

Hygiena [HygienaTV]. (2014a, February 18). MicroSnap Total - 7 hour Total Viable Count! Instructional Demo [Video File]. Retrieved from https://www.youtube.com/watch?v=RqyNv8 EMYPU

Hygiena [HygienaTV]. (2014b, February 20). Hygiena EnSURE \& SystemSURE Plus Quick Start Guide [Video File]. Retrieved from https://www.youtube.com/watch?v=R9d7Wn $\underline{\text { zFit4\&list }=\text { PL4D2DAF807364115F }}$

Hygiena. (2013b, August). Directions for Use MicroSnap-Coliform and E. coli. Retrieved from

http://www.scigiene.com/pdfs/MicroSnap\%2 0Coliform\%20E\%20coli\%20Insert.pdf

Hygiena. (2013c). SystemSURE Plus ATP Cleaning Verification System: Establishing RLU Pass/Fail Limits. Retrieved from http://www.hygiena.com/cat view/22-techdocs/24-healthcare-tech-docs

Hygiena. (2013d). Monitoring Hand Hygiene Using the Hygiena ATP Cleaning Verification System. Retrieved from http://www.hygiena.com/cat_view/22-techdocs/24-healthcare-tech-docs 
Hygiena. (2013e). Guide to ATP Hygiene Monitoring.

Hrudey, S.E., Huck, P.M., Payment, P., Gillham, R.W., \& Hrudey, E.J. (2002). Walkerton: Lessons learned in comparison with waterborne outbreaks in the developed world. Journal of Environmental Eng. Science, 1, 397-407. doi: 10.1139/S02-031

Inside Walkerton: Canada's worst-ever E. coli contamination. (2010, May 10). CBC News. Retrieved October 14, 2014, from http://www.cbc.ca/news/canada/insidewalkerton-canada-s-worst-ever-e-colicontamination- 1.887200

Jagadeesan, Y., Deepa, M., \& Kannagi, M. (2013). Mobile phones as fomites in miocrobial dissemination. International Journal of Current Science, 5, 6-14.

Kramer, A., Schwebke, I., \& Kampf, G. (2006). How long do nosocomial pathogens persist on inanimate surfaces? A systematic review. BMC Infectious Diseases, 6(130), 1-2.

Lopez, G., Kitajima, M., Havas, A., Gerba, C., \& Reynolds, K. (2014). Evaluation of a Disinfectant Wipe Intervention on Fomite-toFinger Microbial Transfer. Applied and Environmental Microbiology, 80(10), 31133118. doi: 10.1128/AEM.04235-13.

Meighan, P. (2014). Validation of the MicroSnap Coliform and E. coli Test System for Enumeration and Detection of Coliforms and E. coli in a Variety of Foods. Journal of AOAC International, 97(2), 453-478. doi:10.5740/jaoacint.13-361

Microbial Guidelines for Ready-to-Eat Foods A Guide for the Conveyance Industry and Environmental Health Officers (EHO). (2010). Health Canada Environmental
Health Bureau Travelling Public Program, $1-7$.

Shaughnessy, R. J., Cole, E. C., Moschandreas, D., \& Haverinen-Shaughnessy, U. (2013). ATP as a Marker for Surface Contamination of Biological Origin in Schools and as a Potential Approach to the Measurement of Cleaning Effectiveness. Journal of Occupational and Environmental Hygiene, 10, 336-346. doi: $10.1080 / 15459624.2013 .784633$

Sifuentes, L., Koenig, D., Philips, R., Reynolds, K., \& Gerba, C. (2014). Use of Hygiene Protocols to Control the Spread of Viruses in a Hotel. Food Environmental Virology, 6, 175-181. doi: 0.1007/s12560-014-9158-0

Srikanth, P., Rajaram, E., Sudharsanam, S., Lakshmanan, A., Mariappan, U., \& Jagannathan, K. (2010). Mobile phones: Emerging threat for infection control. Journal of Infection Prevention, 11(3), 8790.

Tekerekoğlu, M., Duman, Y., Serindağ, A., Cuğlan, S., Kaysadu, H., Tunc, E., \& Yakupogullari, Y. (2011). Do mobile phones of patients, companions and visitors carry multidrug-resistant hospital pathogens? American Journal of Infection Control, 39, 379-381.

Ustun, C., \& Cihangiroglu, M. (2012). Health Care Workers' Mobile Phones: A Potential Cause of Microbial Cross-Contamination Between Hospitals and Community. Journal of Occupational and Environmental Hygiene, 9, 538-542. doi: $10.1080 / 15459624.2012 .697419$ 